\title{
Scrub typhus in Sri Lanka - beyond the stethoscope
}

\author{
Premaratna $\mathbf{R}^{1}$ \\ Journal of the Ceylon College of Physicians, 2017, 48, 61-65
}

\section{Introduction}

Scrub typhus, or tsutsugamushi disease, is an acute febrile illness in humans caused by infection with Orientia tsutsugamushi (OT) following a bite of an infected mite vector of the genus Leptotrombidium ${ }^{1}$. Scrub typhus is endemic in the Asia-Pacific region, extending from Afghanistan to China, Korea, the islands of the western Pacific and Indian Oceans, and northern Australia ${ }^{2,3}$. This endemic region is often referred to as the tsutsugamushi triangle, and hosts approximately 1 billion people 4 . The vectors can be found in a variety of ecological conditions, from the mountainous regions of northern India to the tropical climates of the Malay Peninsula and Indonesia ${ }^{2}$. Trans-ovarial transmission of OT within vectors appears to be essential to maintenance of the agent in nature; thus, the mite serves as both the vector and the reservoir ${ }^{2}$. Transmission of the etiologic agent to vertebrate hosts occurs during feeding of the larval or "chigger" stage of mites $^{2}$. While Orientia is vertically maintained in Leptotrombidium mite populations, it may be transmitted horizontally from mites to vertebrate hosts ${ }^{2}$. The transmission to humans is incidental. Currently, there is no vaccine against scrub typhus ${ }^{5}$.

Scrub typhus became more familiar during World War II, when soldiers deployed to endemic regions were affected in great numbers ${ }^{2}$. Scrub typhus was considered the most devastating and threatened ground attack by an unseen enemy during the world wars, carrying high morbidity and mortality. However, the incidence of scrub typhus died down after the introduction of tetracyclines and chloramphenicol. Similar to other countries in the tsutsugamushi triangle where scrub typhus is endemic the documented history of scrub typhus in Sri Lanka dates back to the Second World War ${ }^{5}$. However, its interest re-emerged during the last few decades due to increasing incidence of the illness. During the past decade we found patients presenting with serious complications such as encephalitis, myocarditis, pneumonitis and multi-organ involvement $6,7,8,9,10,11,12$. The incidence of the disease seems to be based on human activity, and ecological

\footnotetext{
${ }^{1}$ Department of Medicine, Faculty of Medicine, University of Kelaniya, Sri Lanka.
}

and climatic factors. Research since World War II has highlighted dramatic antigenic variation among strains of $\mathrm{OT}^{2}$. Today, more than 20 antigenically distinct strains of OT have been reported, including the initially serologically distinguished prototypic strains Karp, Gilliam, and Kato ${ }^{13}$. The disease mimics several other tropical febrile illnesses, and can vary from mild to fatal disease, with reported mortality rates of $35 \%-50 \%$ during the pre-antibiotic era ${ }^{14,15}$ to $7-9 \%$ currently, in different geographical regions ${ }^{5}$. Detection of the eschar; the site of the bite of the mite assists in the early diagnosis and treatment, thus preventing development of serious complications. However the occurrence of this sign is highly variable and depends on the host, geographical region, and possibly the bacterial strain ${ }^{16}$. Furthermore, great inter-strain variability in virulence has been shown in mouse models. However, it is not clear whether virulence for mice can be directly applicable to humans ${ }^{2}$. Although, antigenic variation may result in a spectrum from very mild to fatal disease ${ }^{2}$, the mortality due to scrub typhus can be reduced dramatically by early diagnosis and treatment ${ }^{17,18,19}$. Although relapses and reinfections are known to occur ${ }^{20}$ this is poorly investigated. However such relapses are also likely to respond to treatment with current antirickettsial antibiotics such as doxycycline and azithromycin².

Until recently the diagnosis of these infections were mainly on clinical grounds and scrub typhus was supported by the Weil Felix test; a test considered non-specific and obsolete today. Therefore in June 2008, in collaboration with the Centers for Disease Control and Prevention (CDC), Atlanta, Georgia, USA, we established IFA based rickettsial disease diagnostics in Sri Lanka. Our initial collaborations with CDC identified Gilliam, Karp, and Kato serotypes as the dominant serotypes in Sri Lanka similar to that is documented in many other geographical locations. Therefore antigens of these serotypes were employed for the disease confirmatory IFA test in Sri Lanka. The IFA-IgG titre of 1:128 was recommended as the diagnostic cut off titre for acute rickettsioses including that for scrub typhus by the CDC as for other endemic regions. Although IFA test was considered the gold standard test for the diagnosis of rickettsial infections, we realised that the CDC recommended cut off titre of 1:128 was not helping the diagnosis of acute rickettsial 
infections in Sri Lanka. This was later identified as due to high background sero-prevalence of $\lg G$ antibodies against scrub typhus and spotted fever group (SFG) rickettsioses in many geographic locations of the country. Although various combinations of antigenically distinct dominant serotypes and genotypes have been increasingly documented in regional countries, we were not aware of the prevailing $O$ tsutsugamushi serotypes and genotypes in Sri Lanka. Identification of serotypes and genotypes of OT circulating in Sri Lanka is considered important in order to improve the diagnostic accuracies as well as for development of vaccines against scrub typhus.

The current IFA based diagnostics against scrub typhus is time consuming, expensive and require trained experts for carrying out the diagnostic procedures and for interpretation. Furthermore, it is impossible for accurate diagnosis when a new or unknown serotype is found ${ }^{20,21}$. Therefore, it was needed to identify Orientia tsutsugamushi serotypes and genotypes in Sri Lanka and to identify or develop a rapid diagnostic test such as immune-chromatographic card tests that could be utilised bed side in the diagnosis of scrub typhus in Sri Lanka.

\section{Methods, results and discussion}

In order to address the problem encountered with IFA-lgG cutoff titres in the diagnosis of acute rickettsial infections in Sri Lanka, the clinical and laboratory database of Rickettsial Disease Diagnostic and Research Laboratory (RDDRL), Faculty of Medicine, University of Kelaniya, was retrospectively analyzed in relation to the known duration of illness at the time of sampling and whether the patients responded favourably to anti-rickettsial antibiotics. Out of 478 samples that had been analysed for rickettsioses by November 2010, the date of collection in relation to illness and follow up serology was known in 261. Fifty six had been sent on or before the 7th day of illness with a mean of 5 days, and 205 were sent after the 7th day with a mean of 19 days. Of the 146 suspected SFG infections, only 3 responders of 25 patients had titres $\leq 1 / 128$ with less than 7 days of illness while all 9 with titres $\geq 1 / 256$ responded (false negative with $1 / 256$ cut-off was $12 \%$, false positive was $0 \%$ ). For illness that was greater than 7 days, the false negative and positive rates at $\geq 1 / 256$ were $5 \%(3 / 59)$ and $11.3 \%$ (6/53). For the 115 suspected ST infections false negative and positive rates with $\geq 1 / 256$ cut-off at less than 7 days of illness were (2/14) and $0 \%(0 / 8)$ respectively while with illness greater than 7 days, false negative and positive rates were $2 \%(1 / 51)$ and $0 \%(0 / 42)$. Therefore, we concluded that in an endemic setting, when the sample is obtained $>7$ days of illness, a single $\geq 1 / 256$ titre is diagnostic for all scrub typhus (ST) infections and $90 \%$ of SFG infections. However, for either SFG or ST, if the sample is obtained $\leq 7$ days of illness, an IgG titre of $\leq 1 / 128$ requires a follow up sample in the diagnosis. Most patients who had a $1 / 128$ titre (the cut-off which is recommended by the CDC in the presumptive diagnosis of acute rickettsioses) in a sample obtained after the 7th day of illness had no clinical rickettsioses ${ }^{22}$.

One drawback in our results was that we were unaware of the spectrum of SFG and ST rickettsial organisms causing disease within our geography and whether higher titres against autochthonous SFG or $\mathrm{ST}$ antigens might be obtained than with $R$. conorii or Orientia tsutsugamushi Karp. This is because it was known that some rickettsial species are known to induce delayed immunological responses and therefore, a delayed rise in $\operatorname{Ig} M$ and IgG titres ${ }^{14,16,18}$. Therefore, we recommended that while initial higher IgG titers against rickettsioses seem to be more accurate in the diagnosis of acute rickettsioses, the need for further studies in order to identify the spectrum of rickettsioses in the country. Consequently, another study to look at concomitant changes in IFA-IgM titres was considered important to clarify uncertainties of mixed IFA-IgG titres against SFG, murine typhus, or $S T$ in the diagnosis of acute rickettsioses in the country. However, cost was the major drawback in additional evaluation of $\operatorname{lgM}$ titres in the diagnosis of rickettsioses in our setting.

With view to address above deficiency and in order to identify the strains of OT causing clinical illness, a preliminary survey was carried out in two scrub typhus prevalent areas along the western coastal belt of Sri Lanka. Ragama and Balapitiya, which are endemic for OT and probably having similar ecological characteristics were selected for this study. Adult patients and children who were admitted with an acute febrile illness and presumed to be having acute scrub typhus based on presence of an eschar and other supporting clinical features were recruited. Eschar biopsies and buffy coat samples collected from patients who were confirmed having OT by IFA were further studied by real time PCR (Orientia $47 \mathrm{kD}$ ) and nested PCR (Orientia $56 \mathrm{kD}$ ) amplification. DNA sequences were obtained for $56 \mathrm{kD}$ gene amplicons and phylogenetic comparisons were analyzed using currently available data in GenBank [Neucleotide substitution per 100 residues, 1000 Bootstrap Trials]. Twenty eschar biopsies (Location 1,19, Location 2,1) and eight buffy coat samples (Location 1,6, Location 2,2 ) examined by real time PCR revealed Orientia amplicons in 16 samples. DNA sequences were obtained for the $56 \mathrm{kD}$ gene amplicons in 12 eschars 
and 4 buffy coat samples. The genotypes of the Location1 samples revealed that, 7 exhibiting close homology with JP1 [distantly related to UT177 Thai (Karp related)], five had close homology with Kato strain, two had close homology with JGv and JG AF [Distantly related to Kawasaki M63383] and one had close homology with Gilliam strain. The Location 2 strain was closely related to Kuroki-Boryong L04956, the genotype which is distributed in far eastern Asia. Similar to other patients in the cohort this patient also had never travelled out of Sri Lanka. This is the first time, the identification of $O$. tsutsugamushi genotypes were carried out in Sri Lanka and we observed all three main OT genotypes in the country, and the majority fell into Thai Karp related clade. These results demonstrate great antigenic diversity of OT in the studied areas of Sri Lanka. However, it is interesting to note the close homology with the Kuroki-Boryong organism in one patient from study site 2 (Ragama) who, like the others, had never travelled out of Sri Lanka² Although we were not in a position to comment on clinical severity of this infection among this small cohort of patients, a 2011 study in Korea comparing the clinical severity between Boryong and Karp genotypes suggested that eschars and rashes were found in $97 \%$ and $94 \%$ of the patients infected with the Boryong cluster compared to $73.7 \%$ and $68.4 \%$ of the patients infected with the Karp cluster, respectively, suggesting clinical variation among infections with different serotypes of $\mathrm{OT}^{24}$. However, in a previous study conducted in the same geographical region, there was a high eschar rate (89\%) that is in keeping with the above findings ${ }^{25}$. Our ongoing studies are further focusing on genotypes of scrub typhus in many other geographical locations in the country.

Accuracy and rapidity of a test to diagnose Orientia tsutsugamushi infection is important in clinical practice which assists early management with appropriate treatment. The clinical diagnosis is complicated with similar clinical symptoms with other acute febrile illnesses including leptospirosis, murine typhus and haemorrhagic fever with renal syndrome. The confirmatory diagnosis of scrub typhus even with the gold standard IFA test is challenging as it has more than twenty serotypes. The identification of high genotypic variability of OT in Sri Lanka and the detection of far eastern serotypes such as KurokiBoryong may suggest probable underperformance of current IFA test which utilizes Karp, Kato and Guillium strains. However, inclusion of antigens in an IFA test to cover all likely serotypes is not feasible. Furthermore, the current IFA test is costly, time consuming and needs expertise in the diagnosis. Therefore, with view to further simplify the diagnosis of scrub typhus in Sri Lankan endemic setting, in collaboration with
Immune Med, Korea, the improved ImmuneMed Scrub Typhus Rapid Diagnostic Test (RDT) was developed and validated for its performance and investigated for its ability to discriminate scrub typhus from seventeen other febrile aetiologies such as hemorrhagic fever with renal syndrome, leptospirosis, and murine typhus ${ }^{26}$.

Based on the fact that $56 \mathrm{kDa}$ of surface antigenic protein of Orientia tsutsugamushi has antigenicity and diagnostic value, genes encoding the fragment of 56 $\mathrm{kDa}$ protein from the major serotypes, including Gilliam (tsg56, GenBank AY335819), Karp (tsa56, GenBank AY956315), and Kato (tst56, GenBank M63382), were amplified by polymerase chain reaction (PCR). The antigenic region was selected from the $56-\mathrm{kDa}$ outer membrane protein gene which showed more than $30 \%$ amino-acid sequence homology with each other at $56-$ $\mathrm{kDa}$ outer membrane proteins of $O$. tsutsugamushi prototype Gilliam, Karp and Kato to make the chimeric $56-k D a$ protein. The amplified DNAs were connected in series and cloned into protein expression vector (pET$22 \mathrm{~b}+)$. The cloned DNA was expressed in E. coli as a fusion protein. This fusion antigenic protein (cr56, 103 $\mathrm{kDa}$ ) which is produced, isolated and purified in a single process can be used in diagnosing scrub typhus.

In addition to Gilliam, Karp, and Kato, the gene encoding 56 kDa protein (kr56, GenBank AF302990) from $O$. tsutsugamushi Kangwon strain and the gene encoding 21 kDa protein (r21, GenBank AM494475) from O. tsutsugamushi Boryong, were amplified by PCR and cloned into protein expression vector (pET30a) to improve the sensitivity of the scrub typhus diagnosis. Each cloned DNA was expressed in E. coli and purified to use in the scrub typhus diagnosis and each protein was added to cr56. The expressed 3 antigens were purified using His-bind Resin (Novagen, Cat No. 69670-4) and dialyzed with potassium phosphate buffer. After formulating each antigen as 2 $\mathrm{mg} / \mathrm{mL}$, the mixed antigens were applied to the test line of the immunochromatographic test.

The sensitivity at the base of each $\lg M$ and $\lg G$ indirect immunofluorescent assay (IFA) in Korean patients was $98.6 \%$ and $97.1 \%$, and the specificity was $98.2 \%$ and $97.7 \%$ respectively. The sensitivity and specificity for retrospective diagnosis at the base of IFA in Sri Lanka was $92.1 \%$ and $96.1 \%$. ImmuneMed RDT was not reactive to any serum from seventeen diseases including hemorrhagic fever with renal syndrome $(n=48)$, leptospirosis $(n=23)$, and murine typhus $(n=48)$. ImmuneMed RDT shows superior sensitivity $(98.6 \%$ and $97.1 \%)$ compared with SD Bioline RDT $(84.4 \%$ at $\lg \mathrm{M}$ and $83.3 \%$ at $\lg G$ ) in Korea. The retrospective diagnosis of ImmuneMed RDT exhibits $94.0 \%$ identity with enzyme-linked 
immuno-sorbent assay (ELISA) using South India patient serum samples. These results suggest that this RDT can replace other diagnostic tests and is applicable for diagnosis of scrub typhus in Sri Lanka. This rapid and accurate diagnosis will be beneficial for diagnosing and managing scrub typhus.

\section{Conclusions}

We conclude that in IFA-lgG based diagnosis of rickettsioses in the endemic setting of Sri Lanka, when the serum sample is obtained $>7$ days of illness, a single $\geq 1 / 256$ titer is diagnostic for all scrub typhus infections and $90 \%$ of SFG infections. However, for either SFG or ST, if the sample is obtained $\leq 7$ day of illness, an $\lg G$ titer of $\geq 1 / 128$ requires a follow up sample in the diagnosis. Most patients who had a $1 / 128$ titer (the cutoff which is recommended by the CDC in the presumptive diagnosis of acute rickettsioses) in a sample obtained after the 7th day of illness had no clinical rickettsioses. For the first time in Sri Lanka we observed all three main OT genotypes in the country, and the majority fell into Thai Karp related clade demonstrating great antigenic diversity of OT in the studied areas of Sri Lanka. However, we also noted the existence of a far eastern serotype Kuroki-Boryong organism in one patient who, like the others, had never travelled out of Sri Lanka suggesting the need for inclusion of such organisms in the diagnostic pool of scrub typhus in the country. For the rapid diagnosis of scrub typhus in the country, the ImmuneMed Rapid Diagnostic test which contained common and far Eastern serotype showed sensitivity and specificity of $92.1 \%$ and $96.1 \%$, and the test kit was not reactive to any serum from seventeen diseases including hemorrhagic fever with renal syndrome, leptospirosis, and murine typhus. These results suggest that this RDT could replace other diagnostic tests and is applicable for diagnosis of scrub typhus in Sri Lanka.

\section{Acknowledgements}

I acknowledge all authors of the three studies who tirelessly worked towards the fruitful outcome of the studies; Weerasinghe S, Ranaweera A, Chandrasena TG, Bandara NW, Dasch GA, de Silva HJ, Blanton LS, Samaraweera DN, de Silva GN, Walker DH, Kim YJ, Park S, Selvaraj S, Park SJ, Kim S, Kim D, Kim MS, Shin DH, Choi KC, Kwon $\mathrm{SH}$, Seo W, Lee NT, Kim SH, Kang HK, Kim YW.

A special word of thank is conveyed to all study participants and their guardians or relatives who consented to provide clinical materials for the three studies and to all overseas collaborators who provided all necessary diagnostics and other required materials free of charge.

I thank Dr. Kumara TADN, Dr. Liyanarachchi MS, Balapitiya Base hospital, Sri Lanka, Dr. Gunasekara C, Dr. Mendis S, Department of Dermatology and the staff Colombo North Teaching Hospital, Ragama,

Laboratory staff of Department of Microbiology, Faculty of Medicine, University of Kelaniya, Rickettsial and Vectorborne Diseases Laboratory of the CDC, Atlanta, Georgia, Department of Internal Medicine, University of Texas Medical Branch, Galveston, Texas, USA. Department of Pathology, University of Texas Medical Branch, Galveston, Texas, USA. The Korea Bank for Pathogenic Viruses, the Biobank of Chonbuk National University Hospital, Biobank of Gyeongsang National University Hospital, Biobank of Keimyung University Dongsan Medical Center and Biobank of Wonkwang University Hospital. The members of the Korea Biobank Network supported by the Ministry of Health, Welfare and Family Affairs.

\section{References}

1. Traub R, Wisseman Jr CL. The ecology of chigger-borne rickettsiosis (scrub typhus). J Med Entomol 1974; 11: 237-303.

2. Kelly DJ, Fuerst PA, Ching WM, Richards AL. Scrub typhus, the geographic distribution of phenotypic and genotypic variants of Orientia tsutsugamushi. Clin Infect Dis 2009; 15(48 Suppl 3): S203-30.

DOI: $10.1086 / 596576$.

3. Oaks Jr SC, Ridgway RL, Shirai A, Twartz JC. Scrub typhus. Inst Med Res Malays Bull 1983; 21: 1-98.

4. Chattopadhyay S, Richards AL. Scrub typhus vaccines, past history and recent developments. Hum Vaccines 2007; 3: 73-80.

5. WHO. Frequently asked questions; Scrub typhus. http,//www.searo.who.int/entity/emerging_diseases/ CDS_faq_Scrub_Typhus.pdf. Accessed on May 1, 2016.

6. Luke $\mathrm{N}$, Munasinghe $\mathrm{H}$, Balasooriya L, Premaratna R. Widespread subcutaneous necrosis in spotted fever group Rickettsioses from the coastal belt of Sri Lanka - a case report. BMC Infect Dis 2017; 17(1): 278.

7. Premaratna R, Wijayalath SH, Miththinda JK, Bandara NK, de Silva HJ. Scrub typhus mimicking Parkinson's disease. BMC Res Notes 2015; 8: 438.

8. Premaratna R, Liyanaarachchi EW, Rajapakse RP, Bandara NK, de Silva HJ. A patient with spotted fever group rickettsiosis mimicking connective tissue disease. Ceylon Med J 2012; 57(3): 127-8.

9. Premaratna R, Ariyaratna N, Botheju WI, Bandara NK, de Silva HJ. Scrub typhus pneumonitis. Int J Infect Dis 2013; 17(4): e284. 
10. Premaratna R, Nawasiwatte BM, Niriella MA, Chandrasena TG, Bandara NK, Rajapakse RP, de Silva HJ. Scrub typhus mimicking enteric fever; a report of three patients. Trans $R$ Soc Trop Med Hyg 2010; 104(4): 309-10. DOI: 10.1016/j.trstmh.2009.08.011. Epub 2009 Sep 17.

11. Premaratna R, Williams HS, Chandrasena TG, Rajapakse RP, Kularatna SA, de Silva HJ. Unusual pancytopenia secondary to haemophagocytosis syndrome in rickettsioses. Trans $R$ Soc Trop Med Hyg 2009; 103(9): 961-3.

12. Premaratna R, ChandrasenaTG, Rajapakse RP, Eremeeva ME, Dasch GA, Bandara NK, de Silva HJ. Rickettsioses presenting as major joint arthritis and erythema nodosum: description of four patients. Clin Rheumatol 2009; 28(7): 867-8.

DOI: 10.1007/s10067-009-1166-3. Epub 2009 Mar 25.

13. Kelly DJ, Richards AL, Temenak J, Strickman D, Dasch GA. The past and present threat of rickettsial diseases to military medicine and international public health. Clin Infect Dis 2002; 34 Suppl 4: S145-69.

14. Smadel JE, Elisberg BL. Scrub typhus rickettsia. In: Horsfall $\mathrm{Jr}$ FL, Tamm I, editors. Viral and rickettsial infections of man. 4th ed. Philadelphia: Lippincott; 1965. p. 1130-42.

15. Kawamura A, Tanaka H. Rickettsiosis in Japan. Jpn J Exp Med 1988; 58: 169-84.

16. Kim DM, Won KJ, Park CY, Yu KD, Kim HS, Yang TY, et al. Distribution of eschars on the body of scrub typhus patients, a prospective study. Am J Trop Med Hyg 2007; 76: 806-9.

17. Smadel JE, Woodward TE, Ley Jr HL, Green R, Mankikar DS. Chloromycetin in the treatment of scrub typhus. Science 1948; 108: 160-1.

18. Smadel JE, Traub R, Ley Jr HL, Philip CB, Woodward TE, Lewthwaite R. Chloramphenicol (Chloromycetin) in the chemoprophylaxis of scrub typhus (tsutsugamushi disease). II. Results with volunteers exposed in hyperendemic areas of scrub typhus. Am J Hyg 1949; 50: 75-91.

19. Smadel JE, Woodward TE, Ley Jr HL, Lewthwaite R. Chloramphenicol (Chloromycetin) in the treatment of tsutsugamushi disease (scrub typhus). J Clin Invest 1949; 28: 1196-215.

20. Elisberg BL, Campbell JM, Bozeman FM. Antigenic diversity of Rickettsia tsutsugamushi: epidemiologic and ecologic significance. J Hyg Epidemiol Microbiol Immunol 1968; 12: 18-25.

21. Jiang J, Marienau KJ, May LA, Beecham HJ 3rd, Wilkinson R, Ching WM, Richards AL. Laboratory diagnosis of two scrub typhus outbreaks at Camp Fuji, Japan in 2000 and 2001 by enzyme-linked immunosorbent assay, rapid flow assay, and Western blot assay using outer membrane 56-kD recombinant proteins. Am J Trop Med Hyg 2003; 69: 60-6.

22. Premaratna R, Weerasinghe $S$, Ranaweera $A$, Chandrasena TG, Bandara NW, Dasch GA, de Silva HJ. Clinically helpful rickettsial disease diagnostic IgG titers in relation to duration of illness in an endemic setting in Sri Lanka. BMC Res Notes 2012; 5: 662.

DOI: 10.1186/1756-0500-5-662. PMID: 23198969

23. Premaratna R, Blanton LS, Samaraweera DN, de Silva GN, Chandrasena NT, Walker DH, de Silva HJ. Genotypic characterization of Orientia tsutsugamushi from patients in two geographical locations in Sri Lanka. BMC Infect Dis 2017; $17(1): 67$.

DOI: 10.1186/s12879-016-2165-z. PMID: 28086810

24. Kim DM, Yun NR, Neupane GP, Shin SH, Ryu SY, Yoon HJ. Differences in clinical features according to Boryoung and Karp genotypes of Orientia tsutsugamushi. PLoS One 2011; 6: e22731.

DOI:10.1371/journal.pone.0022731. Epub 2011 Aug 15.

25. Premaratna R, Loftis AD, Chandrasena TG, Dasch GA, de Silva HJ. Rickettsial infections and their clinical presentations in the Western Province of Sri Lanka, a hospital-based study. Int J Infect Dis 2008; 12: 198-202. Epub 2007 Sep 27.

26. Kim YJ, Park S, Premaratna R, Selvaraj S, Park SJ, Kim S, Kim D, Kim MS, Shin DH, Choi KC, Kwon SH, Seo W, Lee NT, Kim SH, Kang HK, Kim YW. Clinical Evaluation of Rapid Diagnostic Test Kit for Scrub Typhus with Improved Performance. J Korean Med Sci. 2016; 31(8):1190-6. DOI: 10.3346/jkms.2016.31.8.1190. Epub 2016 Jun 7. 